\title{
Effects of eplerenone on resistance to antihypertensive medication in patients with primary or secondary hyperaldosteronism
}

\author{
J. David Spence ${ }^{1}$, Chrysi Bogiatzi ${ }^{1,2}$, Mariya Kuk ${ }^{1,3}$, George K. Dresser ${ }^{4}$, \\ Daniel G. Hackam ${ }^{1,4,5}$ \\ 'Stroke Prevention \& Atherosclerosis Research Centre, Robarts Research Institute, \\ Western University, London, Canada; \\ ${ }^{2}$ McMaster University, Hamilton, Canada; \\ 3University of Ottawa, Ottawa, Canada; \\ ${ }^{4}$ Division of Clinical Pharmacology, Western University, London, Canada; \\ ${ }^{5}$ Department of Epidemiology \& Biostatistics, Western University, London, Canada
}

Address for Correspondence:

Dr. J. David Spence, Stroke Prevention \& Atherosclerosis Research Centre,

Robarts Research Institute, Western

University, 1400 Western Road, London,

ON N6G 2V4, Canada.

Email: dspence@robarts.ca

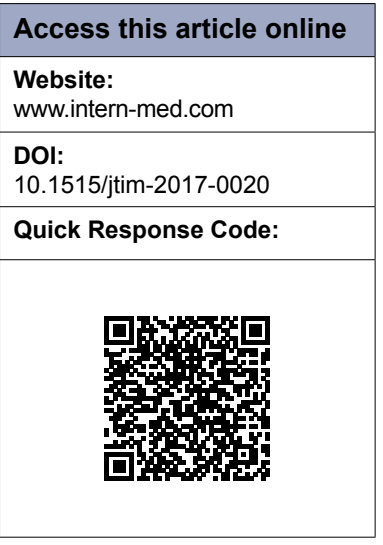

\section{ABSTRACT}

Background and Objectives: Resistant hypertension is an important problem; nearly half of diagnosed hypertensives are not controlled to target blood pressure levels, and approximately $90 \%$ of strokes occur among patients with resistant hypertension. Primary aldosteronism accounts for approximately $20 \%$ of resistant hypertension, but the role of secondary hyperaldosteronism in resistant hypertension is seldom considered. We assessed the effects of eplerenone in patients with hypertension and either primary or secondary hyperaldosteronism. Methods: Patients with a history of resistant hypertension and a supine plasma aldosterone level $\geq 360 \mathrm{pmol} / \mathrm{L}$ were randomized to eplerenone versus placebo in a fully blinded study for one year. A medication intensity score was developed to assess the resistance of hypertension to medication (blood pressure $\times$ medication intensity). We assessed the effects of eplerenone on blood pressure and on resistance to concomitant medication. Results: Final results were available in 37 patients (19 on eplerenone and 18 on placebo). Resistance to medication, as assessed by the intensity of concomitant medication required to maintain blood pressure control, was markedly reduced by eplerenone: medication intensity scores declined by $-0.50 \pm 1.04$ (SD) on placebo versus $-2.11 \pm 1.45$ with eplerenone $(P=0.0001)$, the Systolic Resistance Score declined by $-80.00 \pm 122.93$ on placebo versus $-334.05 \pm 21.73$ on eplerenone $(P=0.0001)$, and the Diastolic Resistance Score increased by $1.28 \pm 31.65$ on placebo and declined by $-40.74 \pm 57.08$ on eplerenone $(P=0.009)$. Conclusions: Eplerenone significantly reduced resistance to concomitant antihypertensive medication in both primary and secondary hyperaldosteronism.

Key words: aldosterone, eplerenone, resistant hypertension, hyperaldosteronism

\section{INTRODUCTION}

Resistant hypertension is an important clinical problem. Despite intensive efforts by national education programs, approximately half of patients diagnosed with hypertension still have systolic blood pressures above $140 \mathrm{mmHg}$, and approximately $20 \%$ still have diastolic pressures above $90 \mathrm{mmHg} .{ }^{[1]}$ Approximately $90 \%$ of strokes occur among persons with uncontrolled hypertension. ${ }^{[2]}$ Resistant hypertension is likely to become more prevalent with the recent publication of the
Systolic Blood Pressure Intervention Trial (SPRINT), ${ }^{[3]}$ which will probably result in lowering of blood pressure targets.

Reasons for poor levels of blood pressure control include therapeutic inertia (reluctance of patients to take medication or increase medication, unjustified belief that white coat hypertension is benign, ${ }^{[4]}$ preoccupation with other health problems with which patients present) and diagnostic inertia (failure of physicians to identify the underlying cause of the hypertension so that appropriate therapy can be prescribed). ${ }^{[5]}$ 
Diagnostic inertia is a particular problem among AfricanAmericans: despite being more likely to have their hypertension diagnosed, more likely to have it treated, and more likely to have it treated more intensively, their blood pressure is less likely to be controlled, ${ }^{[6]}$ with twice the risk of stroke. Reasons for this disparity probably relate to the genetic causes of salt and water retention: primary aldosteronism is more common in African-Americans, and variants of Liddle's syndrome, a mutation of the renal tubular sodium channel, is also probably more common in black patients. ${ }^{[7]}$

In ideal conditions, measuring plasma renin and aldosterone can be used to identify the appropriate therapy: patients with low renin and high aldosterone antagonists are best treated with aldosterone antagonists, and those with low renin and low aldosterone with amiloride. ${ }^{[8]}$ A key issue is that stimulated renin should be measured, as high sodium intakes compress the low normal range, thereby obscuring true suppressed renin activity. ${ }^{[9]}$ However, in the real world, things are not so simple. Patients who begin with low-renin hypertension, once they develop renal damage from hypertension, will develop secondary hyperaldosteronism. Thus aldosterone antagonists may be helpful in resistant hypertension, regardless of the initial cause of the hypertension. Spironolactone has been reported to improve blood pressure in resistant hypertension, ${ }^{[10,11]}$ but is problematic for men because of gynecomastia and mastalgia.

We, therefore, conducted a blinded randomized trial of aldosterone antagonism with eplerenone versus placebo in patients with a history of resistant hypertension and either primary or secondary hyperaldosteronism. In the parent study, we assessed the effects of eplerenone on insulin resistance and on ultrasound measures of carotid wall thickness. In this substudy, we hypothesized that eplerenone would reduce resistance to antihypertensive medications.

In a blinded randomized trial, we analyzed the effect of adding eplerenone versus placebo in patients with hyperaldosteronism on the number and doses of antihypertensive medication and on the resistance to antihypertensive medications. Because participants were continued on concomitant medications, and a target blood pressure was specified, blood pressure per se could not be used to evaluate resistance to medication. We, therefore, developed a Medication Intensity Score and a Hypertension Resistance Score to assess the effect of eplerenone on resistance to concomitant medication.

\section{PATIENTS AND METHODS}

\section{Study population}

Patients being followed in our hypertension clinics (Drs. Dresser and Hackam) and stroke prevention clinics
(Drs. Spence and Hackam) with a history of resistant hypertension that had led to assessment of plasma renin and aldosterone were screened for inclusion based on the previous levels of plasma aldosterone. Plasma aldosterone was repeated at the screening visit to verify eligibility. The number of patients who could be included in the study was limited by the funding from the Heart \& Stroke Foundation of Canada (Ontario).

\section{Inclusion criteria}

Patients with hypertension and either primary or secondary hyperaldosteronism (a supine plasma aldosterone $>360 \mathrm{pmol} / \mathrm{L}$ ) who were being treated medically and willing to provide informed consent were included.

\section{Ethics}

All participants gave written consent to the protocol, which was approved by the Health Sciences Research Ethics Board of the University of Western Ontario (REB \# 17804).

\section{Exclusion criteria}

Excluded were patients with uncontrolled hypertension ( $>160 / 90$ as the mean of 3 measurements seated), renovascular hypertension being considered for bypass or stenting, primary aldosteronism being considered for adrenalectomy, a history of hyperkalemia, symptomatic heart failure, renal failure (eGFR $<50 \mathrm{~mL} / \mathrm{min} / 1.73 \mathrm{~m}^{2[12]}$ ), any condition expected to limit survival to less than 3 years, conditions expected to limit adherence (such as alcoholism, drug addiction, previous known poor adherence to therapy), already taking spironolactone or eplerenone and not willing to be randomized to eplerenone or placebo, and unwilling to consent.

\section{Study medication}

Identical tablets of eplerenone and placebo were kindly provided by the manufacturer (Pfizer).

The starting dose of eplerenone was $25 \mathrm{mg}$ twice daily, increasing to $50 \mathrm{mg}$ twice daily $(2 \times 25 \mathrm{mg}$ tablets twice daily) after one month; matching placebo tablets were provided to participants randomized to placebo. Dose reduction was permitted in cases of hyperkalemia not controlled by withdrawal of other drugs that elevate serum potassium and addition of kaliuretic drugs such as hydrochlorothiazide or furosemide. Dummy dose adjustments were made in some patients randomized to placebo, as determined by the research pharmacy technician.

Randomization was performed using a randomization code generated in SPSS ${ }^{[13]}$; study medication including randomization codes and blinding were coordinated 
between the pharmacy and the registered pharmacy technician working in our unit. Patients and other research personnel were blinded to the treatment assignment.

\section{Concomitant medications}

Patients continued to take their other usual antihypertensive medications, with dose increases as needed to achieve a target clinic blood pressure below $140 \mathrm{mmHg}$ systolic and below $90 \mathrm{mmHg}$ diastolic. Doses of other antihypertensive drugs were reduced if blood pressure declined on addition of eplerenone or placebo; the target blood pressure in both groups was set at $<140 / 90$. The only permitted nonsteroidal anti-inflammatory drug (NSAID) was sulindac, which unlike other NSAIDs, does not impair hypertension control. ${ }^{[14]}$ As is routine in our clinics, participants were given at baseline repeat instructions, educational materials, and recipes to aid them in restricting intake of sodium by eliminating added salt, avoiding salty foods, and following a Mediterranean diet.

\section{Assessment of resistance to concomitant medication}

A Medication Intensity Score was computed to assess the intensity of concomitant medication required to maintain blood pressure control, excluding study medication. This was computed by assigning a dose range (low, medium, high) to each medication being used and then computing the sum of all concomitant medications by dose range. (The dose ranges assigned for each drug are shown in online supplementary material) Thus a participant taking low doses of three different concomitant medications had a score of 3; a participant taking medium doses of three different medications had a score of 6 , a patient taking high doses of three medications had a score of 9, and so on. A Hypertension Resistance Score, reflecting the intensity of medication and the blood pressure achieved by that intensity of medication, was computed by multiplying the blood pressure by the Medication Intensity Score.

\section{Primary aldosteronism versus secondary aldosteronism}

Because the patients were on medication at baseline, including spironolactone and high doses of amiloride in many cases, the plasma renin and aldosterone levels could not be used to classify patients as primary aldosteronism versus secondary aldosteronism by usual criteria of aldosterone-to-renin ratios (ARR). Instead, the ARR were divided into tertiles; the highest tertile of ARR were designated as primary aldo equivalents, the lowest tertile as secondary aldo equivalents, and the remainder as mixed.

\section{Clinical assessments}

Scheduled visits were at baseline, 1 and 2 months for dose adjustment, and at 3, 6, 9, and 12 months. In addition, serum electrolytes were measured at least monthly for safety reasons. (In the EPHESUS trial of eplerenone in heart failure, eplerenone was associated with hyperkalemia in 5\% of patients. $\left.{ }^{[15]}\right)$ Hyponatremia, hypokalemia, or hyperkalemia were treated as clinically indicated. At each visit, blood pressure was recorded as the mean of three readings with a BPTru machine, taken seated after 5 min of rest, in the arm that had the higher blood pressure at the first visit. Blood tests at baseline and end of the study included CBC, electrolytes, urea, creatinine, fasting glucose and insulin, renin, and aldosterone. All tests were performed at the London Health Sciences Centre. Height, weight, and waist circumference were recorded at the first visit; weight and waist circumference at each follow-up visit. Medications and adverse events were recorded at each visit. Extra visits for adverse events including electrolyte abnormalities or inadequate blood pressure control were added as needed.

\section{Plasma renin and aldosterone}

Measurements were performed by methods in routine use in the Biochemistry Department of the London Health Sciences Centre. Details are provided in online supplementary material.

\section{Statistical methods}

Baseline characteristics were described by mean and standard deviation for continuous variables and $n(\%)$ for categorical variables. Changes from baseline to the end of the study in the two treatment groups were assessed by analysis of variance (ANOVA).

\section{RESULTS}

There were 21 participants randomized to placebo and 22 to eplerenone. After dropouts, data after 1 year were available for 37 participants; 19 on eplerenone and 18 on placebo. Baseline characteristics are shown in Table 1. Changes from baseline are shown in Table 2.

As expected, plasma renin and aldosterone increased on eplerenone (Table 2); the change in plasma aldosterone was highly variable and thus was not statistically significant. Serum potassium did not change significantly more with eplerenone than with placebo (Table 2).

\section{Blood pressure}

At baseline, there was no significant difference between the two groups (Table 1). Blood pressure declined during the year of the study; the reduction of diastolic pressure was significantly greater with eplerenone (Figure 1).

\section{Concomitant medication}

At baseline, there was no significant difference in concomitant medication (Table 1); as shown in Table 2, 
Spence et al.: Eplerenone and resistance to antihypertensive medications

\begin{tabular}{|c|c|c|c|}
\hline & Placebo & Eplerenone & Significance \\
\hline & \multicolumn{3}{|c|}{ Continuous variables (Mean $+S D) ; P($ ANOVA) } \\
\hline Age & $68.10 \pm 7.38$ & $69.23 \pm 8.48$ & 0.64 \\
\hline Systolic BP $(\mathrm{mmHg})$ & $129.14 \pm 12.84$ & $135.23 \pm 14.45$ & 0.15 \\
\hline Diastolic BP $(\mathrm{mmHg})$ & $75.86 \pm 7.45$ & $80.45 \pm 8.56$ & 0.07 \\
\hline Heart rate per minute & $65.71 \pm 9.79$ & $64.32 \pm 12.96$ & 0.69 \\
\hline Plasma renin $(\mathrm{ng} / \mathrm{L})$ & $46.21 \pm 80.89$ & $15.0 \pm 9.03$ & 0.08 \\
\hline Plasma aldosterone (pmol/L) & $707.71 \pm 391.39$ & $1,304 \pm 2,077.45$ & 0.20 \\
\hline Serum potassium (mEq/L) & $3.84 \pm 0.55$ & $3.83 \pm 0.41$ & 0.94 \\
\hline Body Mass Index $\left(\mathrm{kg} / \mathrm{m}^{2}\right)$ & $54.19 \pm 9.72$ & $51.76 \pm 10.42$ & 0.43 \\
\hline Smoking (pack-years) & $9.07 \pm 11.72$ & $14.95 \pm 20.14$ & 0.28 \\
\hline Medication score & $5.57 \pm 2.90$ & $5.32 \pm 2.28$ & 0.75 \\
\hline Systolic Hypertension Resistance Scale & $725.95 \pm 389.87$ & $726.09 \pm 364.52$ & 0.99 \\
\hline \multirow[t]{2}{*}{ Diastolic Hypertension Resistance Scale } & $424.90 \pm 228.48$ & $430.27 \pm 215.68$ & 0.94 \\
\hline & \multicolumn{3}{|c|}{ Categorical variables $n(\%) ; P$ Chi Square } \\
\hline Male & $16(76.2 \%)$ & $19(86.4 \%)$ & 0.32 \\
\hline \multicolumn{4}{|l|}{ Smoking status } \\
\hline Never smoked & $8(40 \%)$ & $9(40.9 \%)$ & 0.57 \\
\hline Quit smoking & $11(55 \%)$ & $13(59.1 \%)$ & \\
\hline Still smoking & $1(5 \%)$ & $0(0 \%)$ & \\
\hline Diabetic & $2(9.5 \%)$ & $2(9.1 \%)$ & 0.68 \\
\hline
\end{tabular}

Mean \pm standard deviation; BP: blood pressure.

\begin{tabular}{llll}
\hline \multicolumn{1}{l}{ Table 2: Change from baseline to end of study } & & \\
\hline & Placebo & Eplerenone & $P$ \\
\hline SBP $(\mathrm{mmHg})$ & $-6.11 \pm 13.28$ & $-12.58 \pm 12.25$ & 0.13 \\
DBP (mmHg) & $-1.11 \pm 6.83$ & $-7.42 \pm 7.21$ & 0.01 \\
Medication score & $-0.50 \pm 1.04$ & $-2.11 \pm 1.45$ & 0.0001 \\
Systolic Hypertension Resistance score & $-80.00 \pm 122.93$ & $-334.05 \pm 21.73$ & 0.0001 \\
Diastolic Hypertension Resistance score & $1.28 \pm 31.65$ & $-40.74 \pm 57.08$ & 0.009 \\
Plasma renin & $-10.93 \pm 25.37$ & $26.34 \pm 48.10$ & 0.006 \\
Plasma aldosterone & $-49.06 \pm 341.47$ & $292.57 \pm 1342.63$ & 0.30 \\
Serum potassium (mEq/L) & $0.13 \pm 0.49$ & $0.18 \pm 0.56$ & 0.77 \\
\hline
\end{tabular}

Mean \pm standard deviation

concomitant medication declined significantly among participants randomized to eplerenone. Concomitant medications at baseline and at the end of the study are listed in online supplementary material.

\section{Blood pressure severity/resistance to therapy}

Blood pressure resistance to therapy was not different at baseline (Table 1), but improved significantly on eplerenone (Table 2), as indicated by the intensity of medication needed to achieve the observed systolic and diastolic pressures. Systolic blood pressure changed by $-6.11 \pm 13.28$ $\mathrm{mmHg}$ on placebo versus $-12.58 \pm 12.25$ on eplerenone $(P=013)$; diastolic blood pressure changed by $-1.11 \pm 6.83$ on placebo versus $-7.42 \pm 7.21(P=0.01)$ on eplerenone. With eplerenone, there was a marked reduction in the intensity of concomitant medication intensity $(P=0.0001)$, the systolic hypertension resistance score $(P=0.0001)$, and the diastolic hypertension resistance score $(P=0.009)$ (Figure 2). There was no difference by tertile of ARR for either systolic or diastolic resistance $(P=0.17$ for both.)

\section{Adverse events}

One patient with diabetic nephropathy was hospitalized with a transient decline in renal function; this was unrelated to eplerenone. A minor adverse event of some kind at any visit was reported by $16(76.2 \%)$ of patients taking placebo and $17(77.3 \%)$ on eplerenone; $P=0.61$. A serum potassium of $>5.5 \mathrm{mEq} / \mathrm{L}$ at any visit occurred in $14.3 \%$ of patients on placebo versus $18.2 \%$ on eplerenone $(P=0.53)$. A serum potassium of $>6 \mathrm{mEq} / \mathrm{L}$ occurred in 1 patient on placebo and 1 patient on eplerenone $(P=0.74)$. Adverse events are listed in online supplementary material; there was no pattern that would suggest a causal relationship to eplerenone.

\section{DISCUSSION}

We found improved blood pressure control and a marked reduction of hypertension resistance with eplerenone, as assessed by the intensity of concomitant medication required to achieve blood pressure control. A novel aspect of our 


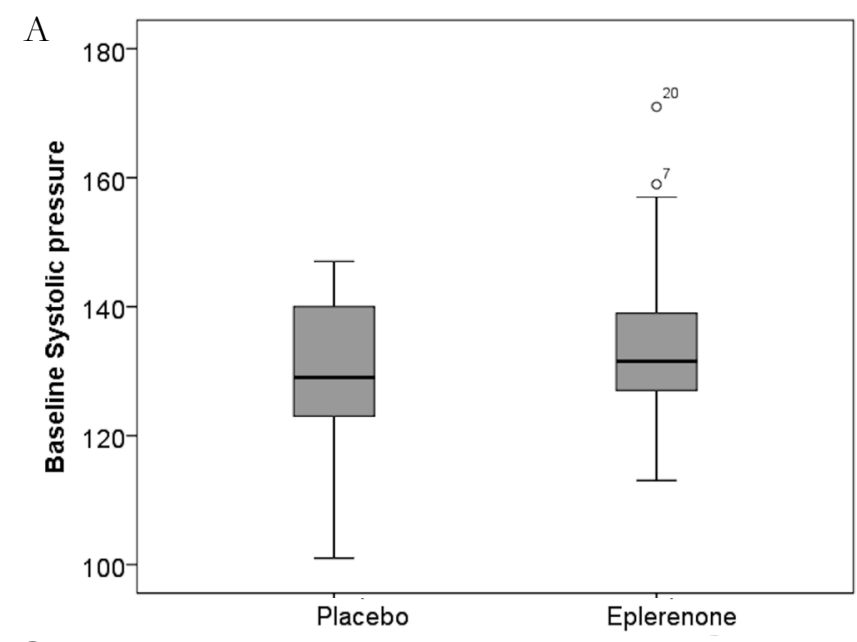

B

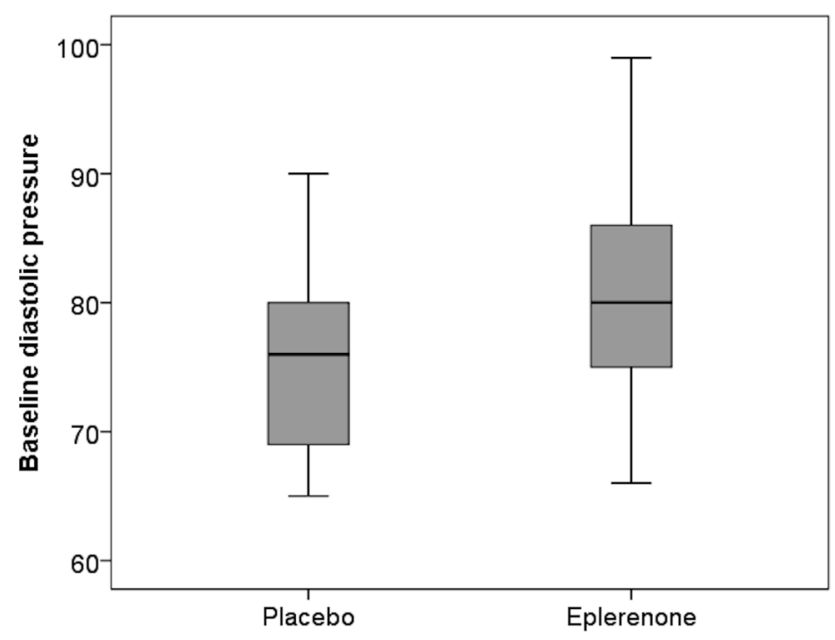

C

$\mathrm{D}$
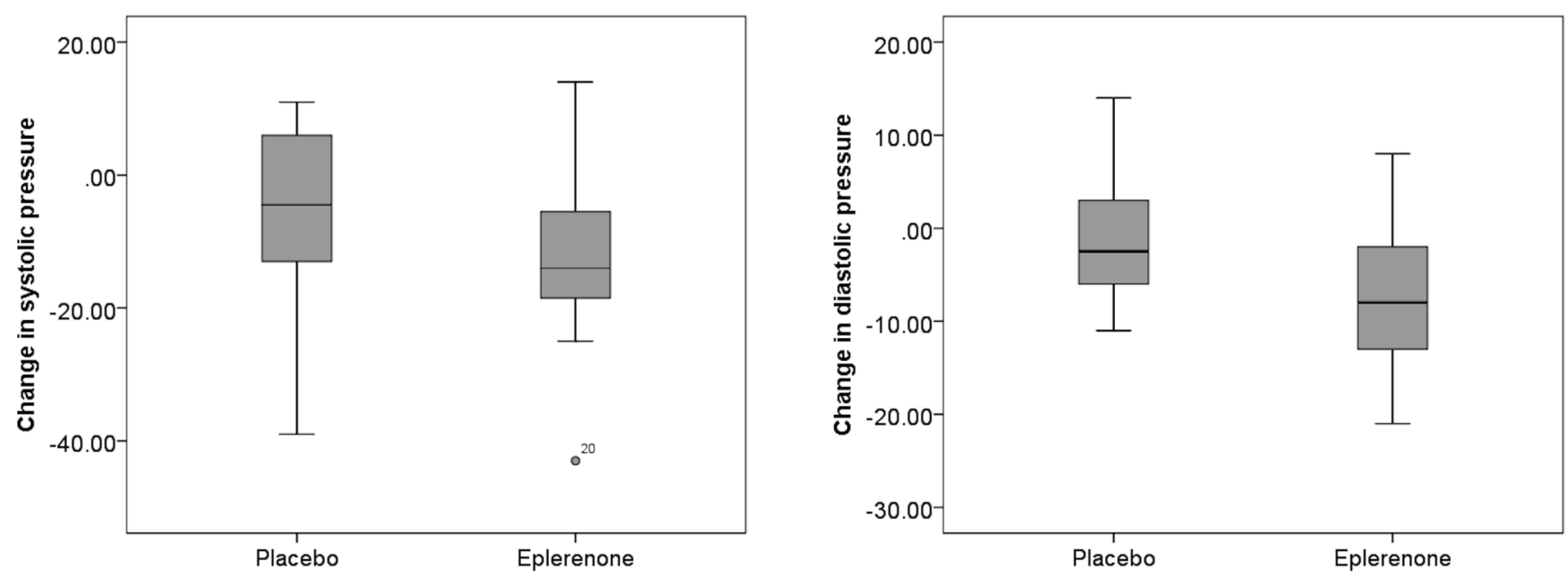

Figure 1: Blood pressure at baseline and changes in blood pressure from baseline to end of the study. Box plots are shown. (A) Baseline systolic pressure, (B) baseline diastolic pressure, (C) change in systolic pressure from baseline to end of study, and (D) change in diastolic pressure from baseline to end of study. Blood pressures were somewhat higher at baseline and declined more by the end of the study in participants randomized to eplerenone. This occurred with a reduction in the concomitant medication in the eplerenone group, as shown in Figure 2 and Table 2.

study was the use of a medication intensity score to compute a score for resistance of hypertension to medication. This score could be used in future studies to define patients with resistant hypertension for genetic studies and studies of strategies to manage resistant hypertension.

An important limitation of the study was the small sample size, which was dictated by the limited funding. Additionally, as shown in the tables, there were important differences between the two study groups, notably with regard to plasma renin, which was much lower in the participants randomized to eplerenone. This suggests that more of the patients on eplerenone had primary aldosteronism. However, as noted earlier, there was no difference in hypertension resistance scores by tertile of ARR.

Our findings suggest that among patients with resistant hypertension, it may be useful to measure plasma aldosterone, with a view to adding an aldosterone antagonist. Although we did not find a significant increase in serum potassium with eplerenone, it would be important to monitor serum potassium after adding an aldosterone antagonist, particularly in patients with impaired renal function.

\section{CONCLUSION}

In patients with either primary or secondary hyperaldosteronism, adding eplerenone reduced the intensity of medication required to control blood pressures.

\section{ACKNOWLEDGMENTS}

Thanks to Katie Ford, the study coordinator, and Dr. Alan Dennis of the Department of Biochemistry at the London Health Sciences Centre for the help with the methods for 
A

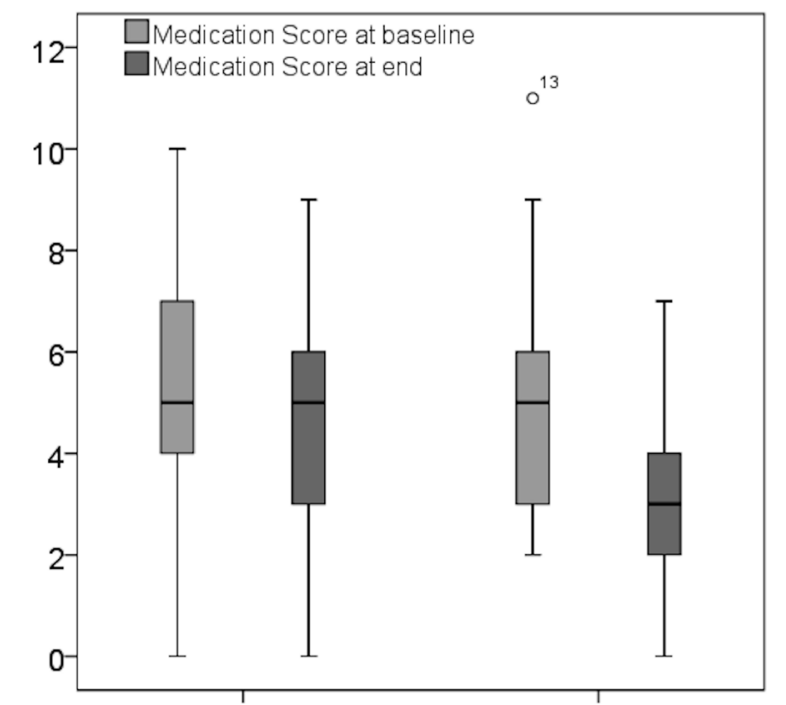

Placebo

Eplerenone

\section{B}

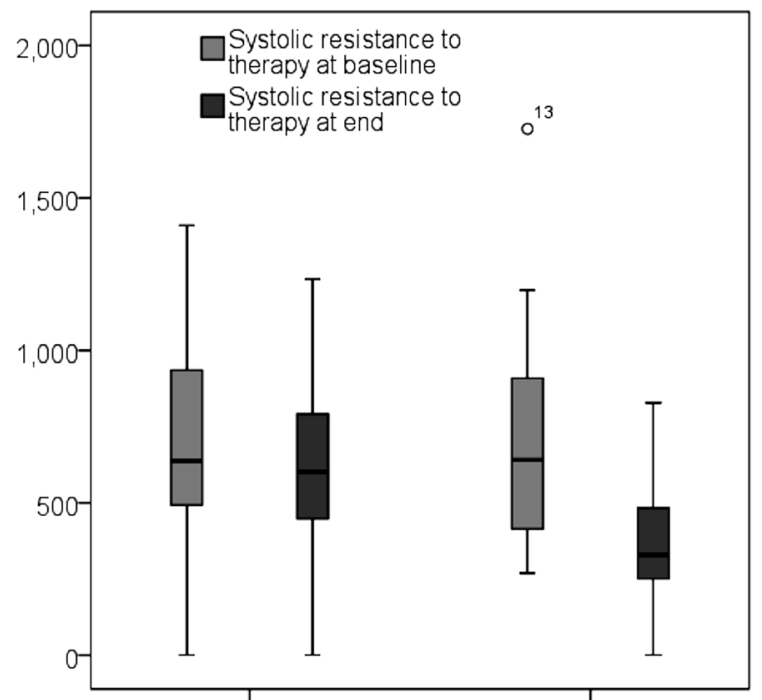

Eplerenone

Placebo

\section{C}

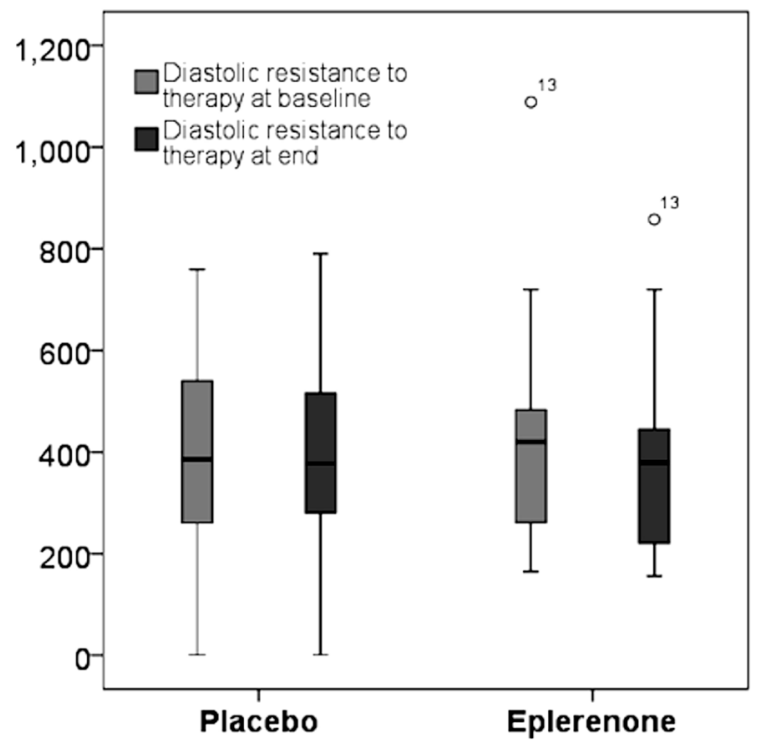

1,000

Diastolic resistance to

therapy at baseline

Figure 2: Resistance to concomitant antihypertensive therapy. Box plots are shown. (A) Change in medication score with placebo versus Eplerenone, (B) change in systolic resistance scale (medication score $\times$ blood pressure), and (C) change in diastolic resistance scale. The intensity of concomitant medication required to maintain blood pressure control, the systolic resistance to medication, and diastolic resistance to medication were all reduced by eplerenone. $P$ values are shown in Table 2.

plasma renin and aldosterone.

\section{Source of Funding}

The study was funded by the Heart \& Stroke Foundation of Canada (Ontario), grant number NA7313. Eplerenone and matching placebo were kindly provided by Pfizer Canada; neither sponsor had any role in the design, analysis, or publication of the results.

\section{Conflicts of Interest}

None of the authors had a conflict of interest related to the topic of this paper.

\section{REFERENCES}

1. Spence JD. Blood pressure control in Canada: the view from a stroke prevention clinic. Can J Cardiol 2015;31:593-5.

2. Li C, Engström G, Hedblad B, Berglund G, Janzon L. Blood pressure control and risk of stroke: a population-based prospective cohort study. Stroke 2005;36:725-30.

3. Sprint RG, Wright JT, Jr., Williamson JD, Whelton PK, Snyder JK, Sink $\mathrm{KM}$, et al. A Randomized Trial of Intensive versus Standard BloodPressure Control. N Engl J Med 2015;373:2103-16.

4. Spence JD. Dilemmas in diagnosing and managing hypertension: is white coat hypertension benign? Can J Cardiol 2015;31:580-2.

5. Spence JD, Rayner BL. J Curve and Cuff Artefact, and Diagnostic Inertia in Resistant Hypertension. Hypertension 2016;67:32-3.

6. Howard G, Prineas R, Moy C, Cushman M, Kellum M, Temple E, et al. Racial and geographic differences in awareness, treatment, and control of hypertension: the REasons for Geographic And Racial Differences in 
Stroke study. Stroke 2006;37:1171-8.

7. Spence JD. Lessons from Africa: the importance of measuring plasma renin and aldosterone in resistant hypertension. Can J Cardiol 2011.

8. Spence JD. Lessons from Africa: the importance of measuring plasma renin and aldosterone in resistant hypertension. Can J Cardiol 2012;28:2547.

9. Wallach L, Nyarai I, Dawson KG. Stimulated renin: a screening test for hypertension. Ann Intern Med 1975;82:27-34.

10. Ouzan J, Perault C, Lincoff AM, Carre E, Mertes M. The role of spironolactone in the treatment of patients with refractory hypertension. Am J Hypertens 2002;15:333-9.

11. Dudenbostel T, Calhoun DA. Use of Aldosterone Antagonists for Treatment of Uncontrolled Resistant Hypertension. Am J Hypertens 2017; 30:103-9.

12. Schwartz MM, Lewis EJ, Leonard-Martin T, Lewis JB, Batlle D. Renal pathology patterns in type II diabetes mellitus: relationship with retinopathy. The Collaborative Study Group. Nephrol Dial Transplant. 1998;13:2547-52.

13. Saghaei M. Random allocation software for parallel group randomized trials. BMC Med Res Methodol 2004;4:26.

14. Wong DG, Spence JD, Lamki L, McDonald JWD. Effect of non-steroidal anti-inflammatory drugs on control of hypertension by beta-blockers and diuretics. Lancet 1986;1:997-1001.

15. Pitt B, Remme W, Zannad F, Neaton J, Martinez F, Roniker B, et al. Eplerenone, a selective aldosterone blocker, in patients with left ventricular dysfunction after myocardial infarction. N Engl J Med 2003;348:130921.

How to cite this article: Spence JD, Bogiatzi C, Kuk M, Dresser GK, Hackam DG. Effects of eplerenone on resistance to antihypertensive medication in patients with primary or secondary hyperaldosteronism. J Transl Intern Med 2017; 5: 93-99. 